\title{
Dynamic study of a compressed electron layer during the hole-boring stage in a sharp-front laser interaction region
}

\author{
W. P. Wang, B. F. Shen, ${ }^{*}$ X. M. Zhang, L. L. Ji, Y. H. Yu, L. Q. Yi, X. F. Wang, and Z.Z. Xu ${ }^{\dagger}$ \\ State Key Laboratory of High Field Laser Physics, Shanghai Institute of Optics and Fine Mechanics, \\ Chinese Academy of Sciences, Shanghai 201800, China
}

(Received 21 January 2012; published 3 August 2012)

\begin{abstract}
This study investigates the dynamics of a compressed electron layer (CEL) when a circularly polarized laser pulse with a sharp front irradiates a high-density foil. A time-dependent model for CEL motion during the hole-boring stage is proposed to describe details of the interaction for any shape of laser pulse. The opacity case, where the laser pulse is totally reflected, is investigated using this model. The results obtained are consistent with the results from particle-in-cell (PIC) simulations. A relaxation distance determined by the laser-front steepness is necessary to build a stable CEL state before ions rejoin into the CEL. For the transparent case, the laser-front steepness is important for the formation of the stable CEL state at the back surface of the target. Considering the motion of ions, both the CEL and ion dynamics are important to rebalance the laser pressure and electrostatic charge-separation force as the hole-boring stage changes to the light-sail stage.
\end{abstract}

DOI: 10.1103/PhysRevSTAB.15.081302

PACS numbers: 52.65.Rr, 52.38.Mf, 52.38.Hb

\section{INTRODUCTION}

The development of laser technology has enabled the present experiments to achieve laser intensities up to the order of $10^{22} \mathrm{~W} / \mathrm{cm}^{2}[1,2]$. High-energy ions accelerated by such intense laser interacting with the solid target can be prospectively applied in fast ignition for inertial confinement fusion [3,4], medical therapy [5,6], and proton imaging [7], among others. Radiation pressure acceleration (RPA) using circularly polarized (CP) laser pulses provides a promising way to obtain a high-energy ion beam with monoenergetic spectrum in a much more efficient manner, compared with target normal sheath acceleration [8-23]. Yan et al. [12] proposed the phase-stable-acceleration (PSA) mechanism in the RPA regime [8-23] to synchronously accelerate and bunch ions within skin depth of the laser pulse to get a monoenergetic ion beam in the longitudinal direction. The laser-front steepness has also been found to be key in obtaining a stable monoenergetic beam in the relativistic region [23]. In these cases, formation of a stable compressed electron layer (CEL) at the back side of the foil is important. Therefore, investigating the dynamics of this CEL is necessary.

Quasiequilibrium on the CEL has been previously investigated [12], and two specific balance conditions have

\footnotetext{
*Corresponding author.

bfshen@mail.shcnc.ac.cn

Corresponding author.

zzxu@mail.shcnc.ac.cn

Published by the American Physical Society under the terms of the Creative Commons Attribution 3.0 License. Further distribution of this work must maintain attribution to the author(s) and the published article's title, journal citation, and DOI.
}

been studied: that between laser pressure and electrostatic charge-separation field force $[10,12]$ and that in the PSA to prevent the displacement of all electrons from the foil $[12,14]$. These conditions, which have recently been investigated analytically $[16,21]$ and applied in experiments [18], provide static solutions at the end of the hole-boring stage, neglecting the effects of ion motion. The dynamics of the CEL and ions during the hole-boring stage is also important for the stable state formation of the CEL and stable ion acceleration.

This study investigates the CEL dynamics during the hole-boring stage in sharp-front laser interactions. Different from our previous work [23], where a uniform velocity was obtained from the simulation and theoretically used to obtain an average velocity of CEL during hole boring, a time-dependent model is proposed to describe the detailed motion of CEL. This model can be used to investigate interactions for any shape of laser pulse. Both one-dimensional (1D) and two-dimensional (2D) particlein-cell (PIC) simulations are carried out to verify this model. For the first case, the ions are fixed so that one can clearly compare the results from the model and the simulation. This process is reasonable in the case of sharpfront laser interaction because ions cannot catch the CEL until they reach the back side of the target [23], which is different from the cases where ions are driven forward together with the CEL $[22,24]$. The relaxation distance and corresponding relaxation time determined by the laser-front steepness are necessary for the formation of a stable CEL state in the opacity case, where the laser pulse is completely reflected. In the transparent case, the laser penetrates through the foil because the target is very thin. Formation of the stable CEL state depends not only on the laser peak amplitude but also on the laser-front steepness. 
For the second case, ions are allowed to move, the motion of the CEL is consistent with the case of fixed ions before the ions catch the CEL. Laser pressure and electrostatic charge-separation force can be rebalanced when ions rejoin the CEL as the hole-boring stage changes to the light-sail stage.

\section{1D THEORY}

\section{A. Model and equations}

The 1D CEL model in hole boring is shown in Fig. 1(a). The incident laser pulse has been assumed to be completely reflected, that is the reflecting efficiency $\eta=1$ [12]. A dense skin-depth electron layer compressed by the laser pressure and the electrostatic force is built up as the laser pulse illuminates a foil. This skin-depth layer is defined as the CEL. Assuming that ions are fixed, the maximum electrostatic charge-separation field $E_{0}=4 \pi e n_{0} d$ increases with the depletion distance $d, e$ is the electron charge, and $n_{0}$ is the foil density. The total electrostatic force on CEL is $\int_{d}^{l} e E_{0}(l-x) /(l-d) n_{\mathrm{p} 0} d x=e n_{0} E_{0} l / 2$, where the density conservation relationship $n_{\mathrm{p} 0}(l-d)=$ $n_{0} l$ is used [10]. In this equation, $l-d\left(l<l_{0}\right)$ is the temporal thickness of the CEL in the hole-boring stage and $l_{0}$ is the foil thickness. The incident laser pressure in the instantaneous frame of the foil is $(2 I / c) \times$ $\left(c-v_{\mathrm{CEL}}\right) /\left(c+v_{\mathrm{CEL}}\right)$, where $I$ is the laser intensity, $v_{\mathrm{CEL}}$ is the velocity of the CEL surface, and $c$ is the light speed. CEL dynamics can be obtained from the total electrostatic and laser pressure forces.

The variables are normalized as follows to simplify comparisons between theory and simulation:

$$
\begin{gathered}
x^{\prime}=x / \lambda, \quad t^{\prime}=t /(\lambda / c), \quad v^{\prime}=v / c, \quad q^{\prime}=q /(-e), \\
m^{\prime}=m / m_{\mathrm{e}}, \quad E^{\prime}=E /\left(m_{\mathrm{e}} \omega_{\mathrm{L}} c / e\right), \quad n^{\prime}=n / n_{\mathrm{c}},
\end{gathered}
$$

where $x, t, v, q, m, E$, and $n$ are the length, time, velocity, charge, mass, field, and density, respectively. $\lambda$ is the laser wavelength, $\omega_{\mathrm{L}}$ is the laser frequency, $m_{\mathrm{e}}$ is the electron rest mass, and $n_{\mathrm{c}}=\omega_{\mathrm{L}}^{2} m_{\mathrm{e}} / 4 \pi e^{2}$ is the critical density.
In the following, the prime of the normalized variable is canceled, and the relativistic equations of the CEL dynamics can be expressed as

$$
\begin{gathered}
\Delta\left(p_{\mathrm{CEL}}\right)=\left[2 a_{t}^{2} \frac{1-v_{\mathrm{CEL}(t-\Delta t)}}{1+v_{\mathrm{CEL}(t-\Delta t)}}-2\left(\pi n_{0} d_{t-\Delta t}\right)^{2}\right] \Delta t, \\
\Delta\left(p_{\mathrm{CEL}}\right)=p_{\mathrm{CEL}(t)}-p_{\mathrm{CEL}(t-\Delta t)}, \\
v_{\mathrm{CEL}(t)}=p_{\mathrm{CEL}(t)} / \sqrt{p_{\mathrm{CEL}(t)}^{2}+\sigma^{2}}, \\
d_{t}=v_{\mathrm{CEL}(t)} \Delta t+d_{t-\Delta t},
\end{gathered}
$$

where $p_{\mathrm{CEL}}=\sigma \gamma v_{\mathrm{CEL}}$ is the CEL areal momentum normalized by $\pi c^{3} m_{\mathrm{e}}^{2} / \lambda e^{2}, \sigma=n_{0} d$ is the areal density of the foil, $\gamma=\left(1-v_{\mathrm{CEL}}^{2}\right)^{-1 / 2}$ is the relativistic factor, and $\Delta t$ is the time step in the calculation. The amplitude of the incident laser pulse at the CEL surface $\left(x=d_{t-\Delta t}\right)$ depends on time as $a_{t}=\left(a_{0} / t_{\mathrm{up}}\right)\left(t-d_{t-\Delta t}\right)$ for $t-d_{t-\Delta t}<$ $t_{\text {up }}$ and $a_{t}=a_{0}$ for $t-d_{t-\Delta t} \geq t_{\text {up }}$. In this equation, $a_{0}=$ $e E_{\mathrm{L}} / m_{\mathrm{e}} \omega_{\mathrm{L}} c$ is the dimensionless laser peak amplitude, $t_{\text {up }}$ is the rising time of the laser front, and $E_{\mathrm{L}}$ is the laser electric field. Details of the interaction for any shape of the laser pulse can be described by changing the form of $a_{t}$. The first term on the right-hand side of Eq. (2) is the laser pressure and the second term is the electrostatic force. Given proper small values of $\sigma, \Delta t$, and $d_{0}=0$ at the initial time $t=\Delta t$, the CEL motion can be modeled (see Fig. 2).

\section{B. Requirements for the model}

The 1D model described by Eqs. (2)-(5) is valid only for opacity cases $(\eta=1)$. Thus, transparency should be avoided during the hole-boring stage. Two conditions to control the complete reflection of the incident laser pulse are discussed in this study.

The first condition is that the foil thickness should be larger than its skin depth $l_{0}>l_{\mathrm{s}}$, where $l_{\mathrm{s}}=c / \omega_{\mathrm{pe}}, \omega_{\mathrm{pe}}=$ $\sqrt{4 \pi n_{\mathrm{e}} e^{2} / m_{\mathrm{e}}}$, and $n_{\mathrm{e}}$ is the electron density. Electrons are
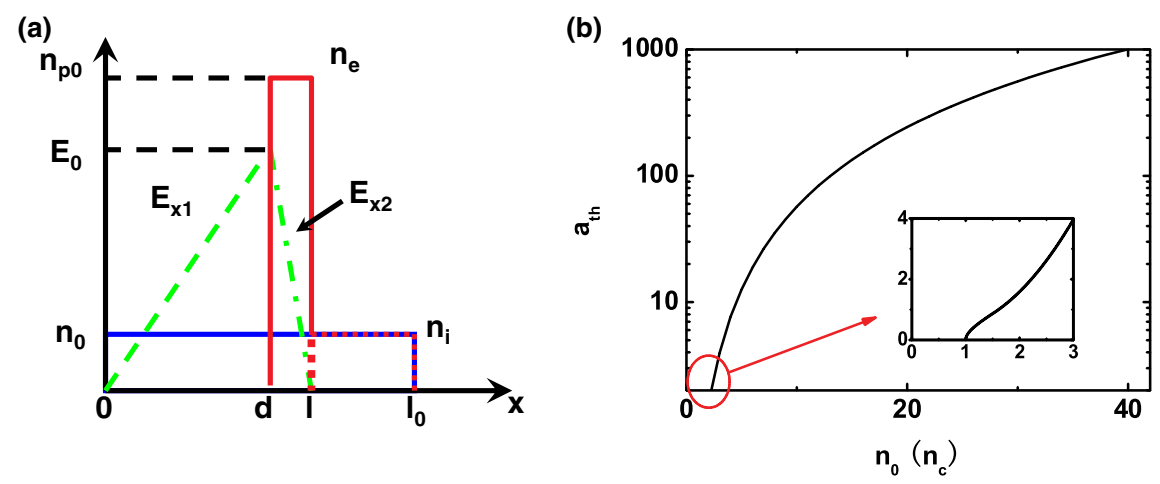

FIG. 1. (a) Sketch of ion density $n_{\mathrm{i}}$ (blue solid line), electron density $n_{\mathrm{e}}$ (red solid line), electrostatic field $E_{x 1}$ (green dashed line), and $E_{x 2}$ (green dash dotted line) in the hole-boring stage. (b) Threshold values of the laser amplitude $a_{\text {th }}$ obtained from Eqs. (13) and (14). 

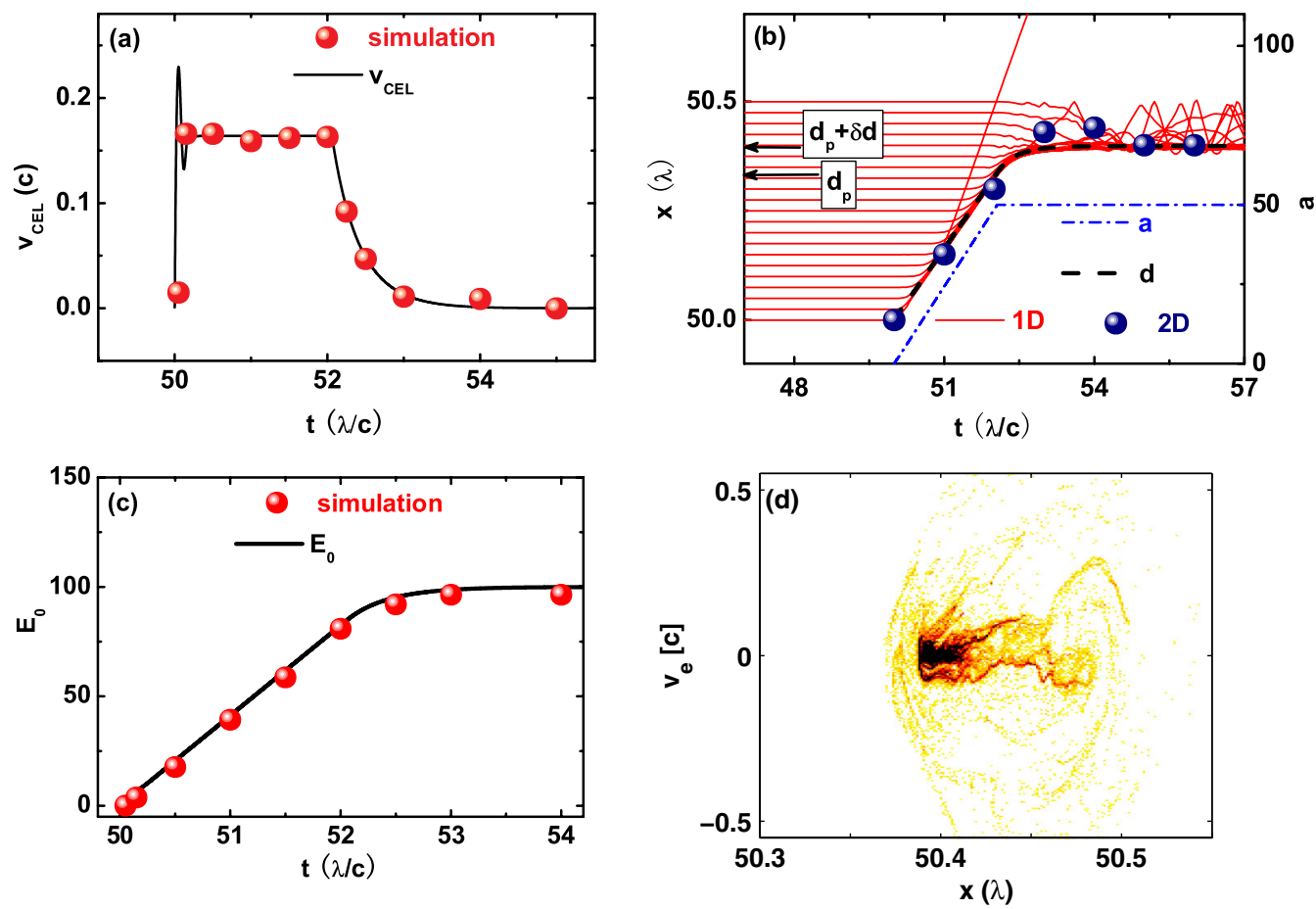

FIG. 2. (a) Velocity of the CEL $v_{\text {CEL }}$ from the analytical model (solid line) and average velocity of the CEL from the simulation (solid circle). (b) CEL positions $d$ (dashed line) and incident laser amplitudes on the CEL surface $a$ (dash dotted line) from the analytical model and electron trajectories (solid lines) from 1D simulation and CEL positions (solid circle) from 2D simulation. (c) Maximum charge-separation electric field $E_{0}$ from the analytical model (solid line) and simulation (solid circle). (d) Phase-space distribution of electrons at $t=55 T$, where $T=\lambda / c$ is the laser period and $\lambda$ is the laser wavelength. Here $n_{0}=40 n_{\mathrm{c}}, l_{0}=0.5 \lambda$, and $a_{0} / t_{\text {up }}=29\left(a_{0}=50, t_{\text {up }}=1.72 T\right)$.

assumed to be completely separated from ions initially to understand the effect of this condition. Neglecting ion motion, electrons (initially at $0<x<\zeta$ ) are assumed to be piled into a thin layer at $x=\zeta$, and ions pile up at $x=0$. In this study, $\zeta$ is defined as the position of the electron layer. The electron layer oscillates around the balance center $(x=0)$, and the equation of motion of the electron layer can be expressed as

$$
\frac{d^{2} \zeta}{d t^{2}}=-\omega_{\mathrm{pe}}^{2} \zeta
$$

Generally, $l_{\mathrm{s}}$ is approximately considered as the largest distance $\zeta_{\max }$ that the oscillating electron layer can reach, that is $l_{\mathrm{s}} \approx \zeta_{\max }$, where $d \zeta / d t=0 . d \zeta / d t>0$ appears at $x=l_{0}$ if $l_{0}$ is much smaller than $l_{\mathrm{s}}$, that is the laser pulse fails to significantly decay within such a thin layer, and all of the electrons will be accelerated by the transmitted laser field. In this case, our 1D model will no longer be valid.

The second condition arises from the relativistic selfinduced transparency effect during the ultraintense laser interaction with the overdense plasma $[8,25,26]$. The incident laser amplitude $a_{0}$ should be smaller than the threshold value $a_{\mathrm{th}}$ in case of complete reflection. Stationary solutions for which the longitudinal electron velocity vanishes have been discussed in Ref. [8]. In this paper, this stationary state also appears in the stage where the laser pulse remains flattop, as shown in Figs. 2(a), 2(b), and 2(d). Equation (7) can be obtained when $d p_{\xi} / d t=0$ :

$$
\partial \psi / \partial \xi=\partial \gamma / \partial \xi=-E_{0} .
$$

In this equation, $\xi=\omega_{\mathrm{L}} x / c$ is defined. $\psi=e \phi / m_{\mathrm{e}} c^{2}$ is the normalized scalar potential and $\gamma=\sqrt{1+a^{2}}$ is the relativistic factor for the incident CP laser pulse with the form $a=a_{0}(\xi) \exp \left[i \omega_{\mathrm{L}} t+i \theta(\xi)\right]$. Two constants of an electron motion in the laser field can be obtained as follows [27]:

$$
M=\left(1-\gamma^{2}\right) \partial_{\xi} \theta
$$

$$
W=\left[\left(\partial_{\xi} \gamma\right)^{2}+M^{2}\right] /\left[2\left(\gamma^{2}-1\right)\right]+\gamma^{2} / 2-n_{0} \gamma .
$$

The following equations can be obtained with the continuity conditions of transverse electric and magnetic fields at the left interface $(x=d)$ :

$$
\begin{gathered}
2\left(a^{2}+a_{\mathrm{r}}^{2}\right)=\left(\partial a_{d} / \partial \xi\right)^{2}+M^{2} / a_{d}^{2}+a_{d}^{2}, \\
M=a^{2}-a_{\mathrm{r}}^{2}, \\
W=|M|+1 / 2-n_{0}(|M|+1)^{1 / 2},
\end{gathered}
$$

where $a_{\mathrm{r}}$ is the amplitude of the reflected wave, and $a_{d}$ is the amplitude at the inner side of the left surface $(x=d)$ [see Fig. 1(a)]. $M=0$ and $W=1 / 2-n_{0}$ were obtained 
for the case of complete reflection $(\eta=1)$. The threshold value of the incident laser amplitude can then be obtained as [8]

$$
\begin{gathered}
a \leq a_{\mathrm{th}}=\sqrt{n_{0}\left(n_{0}-1\right)} \quad\left(1 \leq n_{0} \leq 1.5\right), \\
a \leq a_{\mathrm{th}}=\sqrt{\frac{1}{6}\left(1+a_{d}^{2}\right)\left(2 a_{d}^{2}-n_{0}\right)-\frac{1}{4} a_{d}^{4}} \quad\left(n_{0}>1.5\right),
\end{gathered}
$$

where $\quad a_{d}=\left[\left(3 n_{0}+\sqrt{9 n_{0}^{2}-16 n_{0}+16}\right)^{2} / 16-1\right]^{1 / 2}$. The variation in the ponderomotive force when the laser amplitude $a$ increases from the threshold value $a_{\text {th }}$ to $a_{\mathrm{th}}+a_{1}$ is obtained as

$$
\Delta F_{\mathrm{p}}=-\frac{n_{0} a_{d} / \sqrt{1+a_{d}^{2}}}{\left[2 n_{0}\left(\sqrt{1+a_{d}^{2}}-1\right)-a_{d}^{2}\right]^{1 / 2}} a_{1},
$$

which is negative. The ponderomotive force will be less than the electrostatic charge-separation force [26]. $a_{1}$ is a small amplitude value exceeding the threshold value $a_{\mathrm{th}}$. Electrons are then able to escape from CEL to the vacuum region $(x<d)$. The laser pulse starts to become transparent. Therefore, $a<a_{\mathrm{th}}$ is also required for the 1D model. The threshold value $a_{\text {th }}$ is shown in Fig. 1(b) according to Eqs. (13) and (14).

\section{1D SIMULATIONS AND DISCUSSIONS}

The model from Eqs. (2)-(5) is compared with 1D PIC simulations. A CP laser pulse with the wavelength $\lambda=$ $1 \mu \mathrm{m}$ irradiates the plasma foil. The laser pulse has a trapezoidal profile (linear growth-plateau-linear decrease) in time. The duration is $2 t_{\text {up }}+20 T$ $\left(t_{\text {up }}-20 T-t_{\text {up }}\right)$. Here, $T=\lambda / c$ is the laser period. The peak of the laser amplitude is $a_{0}$. The front side of the foil is located at $x=50 \lambda$. The foil is assumed to be fully ionized to electrons and protons before the laser irradiation at $t=50 T$. The simulation box size is $100 \lambda$ and the mesh size is $\lambda / 200$. Ions are initially fixed to clearly compare the results from model and simulation, which is reasonable because ions cannot catch the CEL until they reach the back side of the target in our case. Ion motion is then taken into account.

\section{A. Dynamics of CEL for thick foil}

First, an opacity case is shown in Fig. 2, where the foil is thick enough and the CEL does not run over the back surface of the foil. A CP laser pulse with a front steepness of $a_{0} / t_{\text {up }}=29\left(a_{0}=50\right.$ and $\left.t_{\text {up }}=1.72 T\right)$ is used. The corresponding peak intensity of laser pulse is $I_{0}=$ $2 a_{0}^{2} \times 1.37 \times 10^{18} \mathrm{~W} / \mathrm{cm}^{2}=6.85 \times 10^{21} \mathrm{~W} / \mathrm{cm}^{2}$. The foil density is $n_{0}=40 n_{\mathrm{c}}$ and its thickness is $l_{0}=0.5 \lambda$.

The average CEL velocity from the simulation is shown in Fig. 2(a) to verify the theoretical model. Except for the initial analytical velocity peak of $0.24 c$, results from the analytical model agree well with the simulation. The CEL velocity $v_{\mathrm{CEL}}=0.164 c$ remains constant when the laser pulse linearly increases. The peak amplitude of the laser pulse $a_{0}=50$ begins at $t=52.06 T$, at which point the corresponding position is $x=d_{\mathrm{p}}=50.34 \lambda$, and then remains constant [see Fig. 2(b)]. The corresponding chargeseparation electric field $E_{x=d_{\mathrm{p}}}=84.83$ [see Fig. 2(c)], where $E_{x}=2 \pi n_{0} d$. After $\delta t=1.94 T$, the maximum of the charge-separation electric field $E_{0}=100$ appears, indicating that CEL can still travel for an additional distance $\delta d$ when the peak amplitude of laser pulse $a_{0}$ remains constant, as shown in Fig. 2(b). $\delta t$ and $\delta d$ are defined as the relaxation time and distance used to build the stable CEL state $\left(v_{\mathrm{CEL}}=0\right)$, respectively, when the peak amplitude of the laser pulse remains constant. $\delta d$ can be calculated by $\int_{0}^{\delta t} v_{\mathrm{CEL}} d t=0.06 \lambda$ based on Eqs. (2)-(5), which is consistent with the simulation result in Fig. 2(b).

After $t=54 T$, the position of the CEL remains constant at about $x=50.4 \lambda$ both in the analytical model and simulation, as shown in Fig. 2(b). Because $d p / d t=0$ in this case, the threshold value of the incident laser $a_{\text {th }}$ determined by Eqs. (13) and (14) can be used. For $n_{0}=40 n_{\mathrm{c}}$, $a_{\mathrm{th}}=1005$ is much higher than the peak amplitude $a_{0}=50$, thus the relativistic self-induced transparency effect can be largely suppressed in our case. In addition, $l_{0}=0.5 \lambda$ is much larger than the skin depth $l_{\mathrm{s}}=0.025 \lambda$ for the initial electron density $n_{0}=40 n_{\mathrm{c}}$, thus the model can be used.

Figures 3(a) and 3(b) show the effects of the laser-front steepness $a_{0} / t_{\text {up }}$ on the CEL velocity and position, respectively. $a_{0}=50$ is kept constant and $a_{0} / t_{\text {up }}$ is changed. Figure 3(b) shows that the CEL arrives at the same stable position $x=50.4 \lambda$ for different $a_{0} / t_{\text {up }}$ mainly because $v_{\text {CEL }}$ decreases to zero when the CEL finally reaches the stable state, as shown in Fig. 3(a). Based on Eq. (2), the balance condition for the stable CEL state $\left(v_{\mathrm{CEL}}=0\right)$ can be expressed as

$$
a_{0}=\pi n_{0}\left(d_{\mathrm{p}}+\delta d\right)
$$

Equation (16) indicates that stable distance $d_{\mathrm{p}}+\delta d$ only depends on the laser peak amplitude $a_{0}$ in this case. Equation (16) transforms to $a_{0}(1+\eta)^{1 / 2} \approx\left(n_{0} / n_{c}\right)\left(l_{0} / \lambda\right)$ for $\eta=1$ in Ref. [12] when the stable state of CEL appears at the back surface of the foil $\left(l_{0}=d_{\mathrm{p}}+\delta d\right)$. For $n_{0}=40$ and $a_{0}=50, l_{0}=0.4$ is obtained. Figure 3(d) shows that CEL can constantly stay at the back surface of the foil in the simulation, which verifies our theoretical expectation.

However, the relaxation distance $\delta d$ varies with $a_{0} / t_{\text {up }}$ for the same $a_{0}$, as shown in Figs. 3(b) and 3(c). The sharper the laser front, the larger the relaxation distance $\delta d$ needed for the formation of the stable CEL state, which is verified by the simulation in Fig. 3(b). This condition has a close relation with the CEL dynamics, such as the CEL velocity. Figure 3(a) shows that the larger constant flattop 

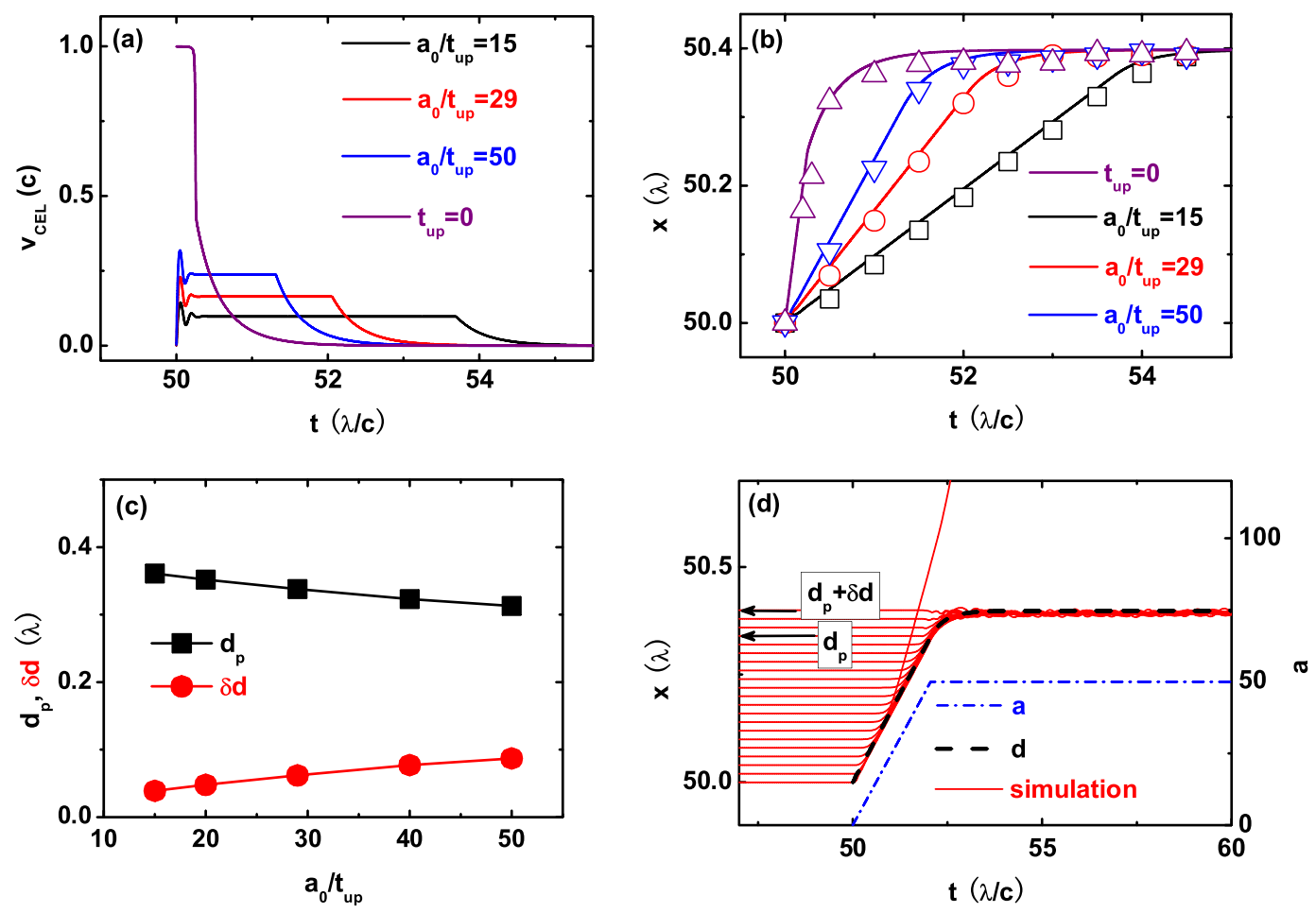

FIG. 3. (a) Velocity of the CEL $v_{\text {CEL }}$ for different laser-front steepness $a_{0} / t_{\text {up }}=15$ (black), 29 (red), 50 (blue), and $\infty$ ( $t_{\text {up }}=0 T$ ) (purple) from the analytical model. (b) CEL positions from the analytical model (solid lines) and simulation (point symbols). (c) Relaxation distance $\delta d$ and the CEL position when the laser peak $a_{0}$ reaches $d_{\mathrm{p}}$ versus $a_{0} / t_{\text {up }}$. Here $n_{0}=40 n_{\mathrm{c}}, l_{0}=0.5 \lambda$, and $a_{0}=50$. (d) CEL positions $d$ (dashed line) and incident laser amplitudes on the CEL surface $a$ (dash dotted line) from the analytical model and electron trajectories (solid lines) from the simulation for $n_{0}=40 n_{\mathrm{c}}, l_{0}=0.4 \lambda$, and $a_{0} / t_{\mathrm{up}}=29\left(a_{0}=50\right.$ and $\left.t_{\text {up }}=1.72 T\right)$.

velocity of CEL can be obtained by a laser pulse with a sharper laser front. For $n_{0}=40, l_{0}=0.5$, and $a_{0} / t_{\text {up }}=$ 29 , the constant velocity $v_{\mathrm{CEL}}=0.164 \mathrm{c}$ is obtained, which is consistent with the theoretical estimation and simulations in Ref. [23]. The constant flattop velocity determined by the laser-front steepness $a_{0} / t_{\text {up }}$ can also be obtained from our time-dependent model [see Fig. 3(a)].

\section{B. Dynamics of the CEL for thin foil}

Based on Fig. 3(b), the stable distance only depends on $a_{0}$ for the same foil, thus $a_{0}=50$ is the unique solution for $d_{\mathrm{p}}+\delta d=0.4 \lambda$. The stable CEL state cannot be built at the back surface of the foil if $l_{0}<0.4 \lambda$ and other parameters are kept similar to those in Fig. 3(d), as shown in Fig. 4(a). The CEL disperses after $t=52.14 T$, and the laser pulse begins to go through the foil. Some electrons run over the back surface of the foil and others are dragged back. The model from Eqs. (2)-(5) is no longer valid after $t=52.14 T$ because the laser pulse is no longer completely reflected [see Fig. 4(a)].

The stable CEL state can be formed again if $a_{0} / t_{\text {up }}$ is deduced from 29 to 1 with other parameters remaining similar to those in Fig. 4(a). Too smooth laser front is not realistic for our model because ions can catch the CEL during the long hole-boring progress. In this study, we only want to show the intrinsic effect of the laser front on the formation of a stable state in the transparent case. Figure 4(b) shows that the CEL arrives at the back surface of the foil at $t=94.7 T$, where $a=44.3$, and then remains constant. Figure 4(c) shows the electric field of the laser pulse $a$ and electron density $n_{\mathrm{e}}$ at $t=120 T$ for $a_{0} / t_{\text {up }}=1$ $\left(a_{0}=50\right.$ and $\left.t_{\mathrm{up}}=50 T\right)$. Electrons can be piled up in a thin layer at $x=0.35 \lambda$; however, part of the laser pulse goes through the foil in this case. Therefore, the model from Eqs. (2)-(5) cannot be used during this stage, as shown in Fig. 4(b). Compared with Figs. 4(a) and 4(b), a stable CEL state can be built again by smoothing the laserfront steepness in the thin foil case, which is mainly attributed to the CEL dynamics during the hole-boring stage. Applying Eqs. (2)-(5), the constant flattop velocities for $a_{0} / t=29$ and 1 can be obtained as $0.164 c$ and $0.008 c$, respectively. The small velocity in the later case leads to small oscillations of the CEL as the hole-boring changes to the light-sail stage, as shown in Fig. 4(b). Therefore, the incident laser pulse can be stably transmitted through the CEL [see Fig. 4(c)]. The amplitude of the transmitted laser pulse $a_{\mathrm{tr}}=\sqrt{M}$ can be theoretically obtained by using Eqs. (7)-(9) [8], as shown in Fig. 4(d). The corresponding simulation results are almost consistent with the theory; however, $a_{\mathrm{tr}}$ is slightly higher than the theoretical result for 

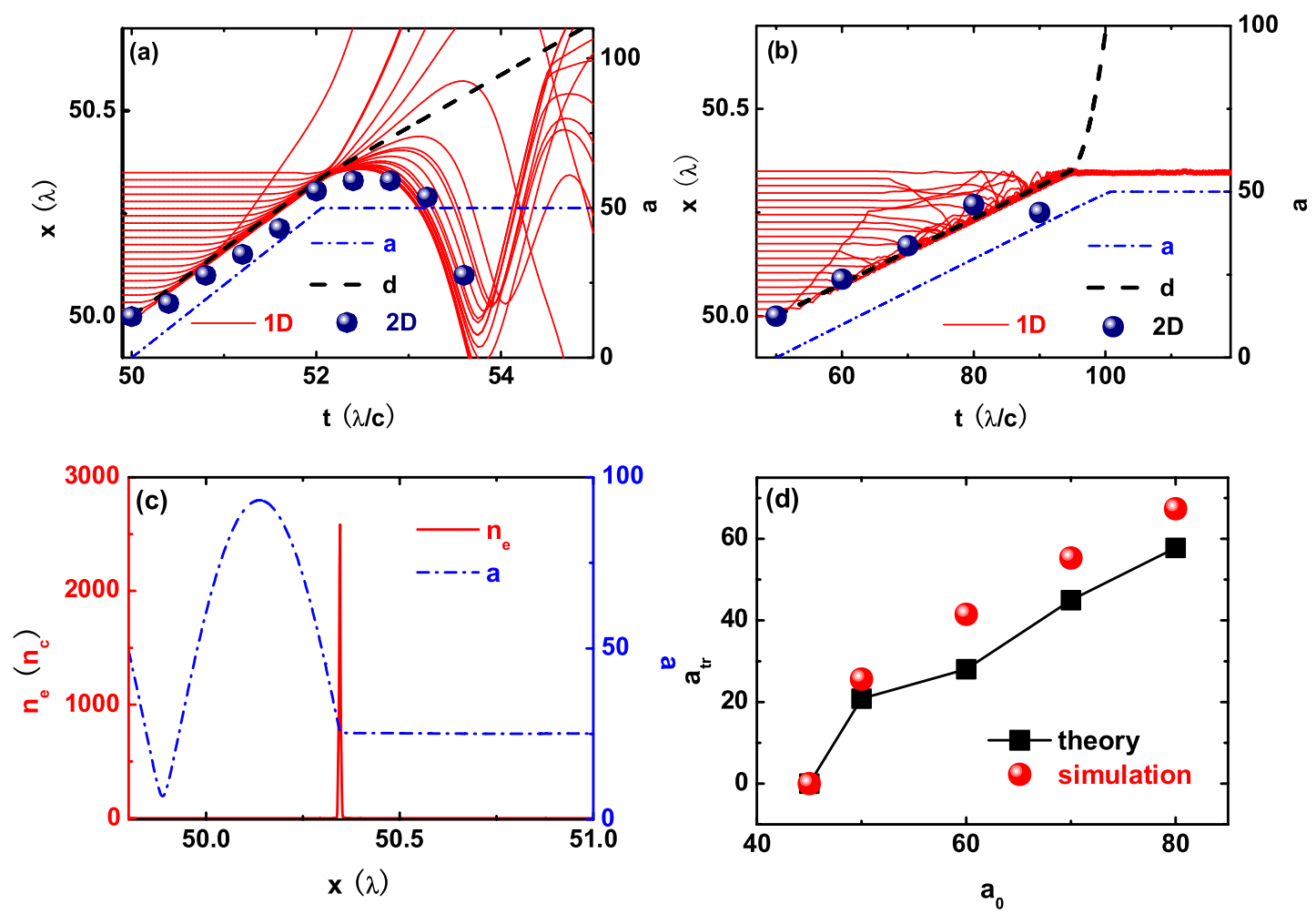

FIG. 4. CEL positions $d$ (dashed line) and incident laser amplitudes on the CEL surface $a$ (dash dotted line) from the analytical model and electron trajectories (solid lines) from 1D simulation and the position of CEL (solid circle) from 2D simulation for (a) $a_{0} / t_{\text {up }}=29\left(a_{0}=50\right.$ and $\left.t_{\text {up }}=1.72 T\right)$ and (b) $a_{0} / t_{\text {up }}=1\left(a_{0}=50\right.$ and $\left.t_{\text {up }}=50 T\right)$. Here $n_{0}=40 n_{\mathrm{c}}, l_{0}=0.35 \lambda$. (c) Electric field of laser pulse $a$ (dash dotted line) and electron density $n_{\mathrm{e}}$ (red solid line) at $t=120 T$. (d) Transmitted amplitude of the laser pulse $a_{\mathrm{tr}}$ versus $a_{0}$ from theory (square) and simulation (circle). Here $a_{0} / t_{\mathrm{up}}=1$ is used for various $a_{0}$.

larger $a_{0}$, which may be reduced to a certain extent by decreasing $a_{0} / t_{\text {up }}$.

\section{Effects of ion motion on CEL formation}

In this section, ions are allowed to move during the holeboring stage. The 1D analytical model proposed above can still be used if the laser front is sharp enough, that is, ions move behind the CEL during the hole-boring stage [23]. In this case, the ion dynamics mainly driven by $E_{x 1}$ can be expressed as

$$
\begin{gathered}
v_{\mathrm{i} 1}=\frac{2 \pi q_{\mathrm{i}} E_{0}}{m_{\mathrm{i}} \gamma_{\mathrm{i}}} \frac{x_{10}}{d} t_{1}, \\
x_{\mathrm{i} 1}=\frac{\pi q_{\mathrm{i}} E_{0}}{m_{\mathrm{i}} \gamma_{\mathrm{i}}} \frac{x_{10}}{d} t_{1}^{2}+x_{10},
\end{gathered}
$$

where $v_{\mathrm{i} 1}$ and $x_{\mathrm{i} 1}$ are the velocities and positions of the ions, respectively, $t_{1}$ is the time $E_{x 1}$ acting on the ions initially resting at $x_{10}\left(0<x_{10}<d\right), q_{\mathrm{i}}$ is the ion charge, and $\gamma_{\mathrm{i}}=\left(1-v_{\mathrm{i} 1}^{2}\right)^{-1 / 2}$. Equations (17) and (18) can be transformed to their unrelativistic forms in Ref. [23] if $\gamma_{\mathrm{i}} \approx 1$.

The motion effect of ions is shown in Fig. 5(a). Different from Fig. 4(a), the CEL can be formed again as ions catch up with electrons [see Fig. 5(a)]. Thus the PSA works well. However, part of the laser pulse now goes through the foil [see Fig. 5(b)] because the CEL slightly disperses after leaving the back surface of the foil. Electrons are heated by the penetration of laser pulses, leaking into the vacuum. Consequently, the PSA model cannot be applied. The fastest ions must initially rest in the middle of the foil and the CEL should arrive at its back surface simultaneously to start a PSA as early as possible in the lightsail stage [23]. Keeping the steepness of the laser front $a_{0} / t_{\text {up }}=29$ and foil density $n_{0}=40$ similar to those in Fig. 5(a), the foil thickness $l_{0}=0.5 \lambda$ is obtained based on Eqs. (2)-(5), (17), and (18), consistent with the theoretical results in Ref. [23]. In this study, the parameters of $a_{0}=96$ and $t_{\text {up }}=3.3 T$ are used. Figure 5(e) shows that the fastest ions and the CEL arrive at the back surface of the foil at about $t=53 T$. The laser pulse is completely reflected by the CEL [see Fig. 5(f)], and the PSA works well in the light-sail stage [see Fig. 5(e)]. This result is identical to that in Ref. [23], where the effects of front steepness $a_{0} / t_{\text {up }}$ on stable ion acceleration are highlighted and discussed in detail.

According to the balance condition $l_{0} \geq\left(1 / 2 \pi c n_{\mathrm{i}}\right) \times$ $\sqrt{\left(I_{0} n_{\mathrm{c}} \lambda\right) /\left(m_{\mathrm{e}} c\right)}$ [14], $a_{0}=96$ is obtained and applied in Figs. 5(c)-5(f), which is larger than $a_{0}=62.8$ for 

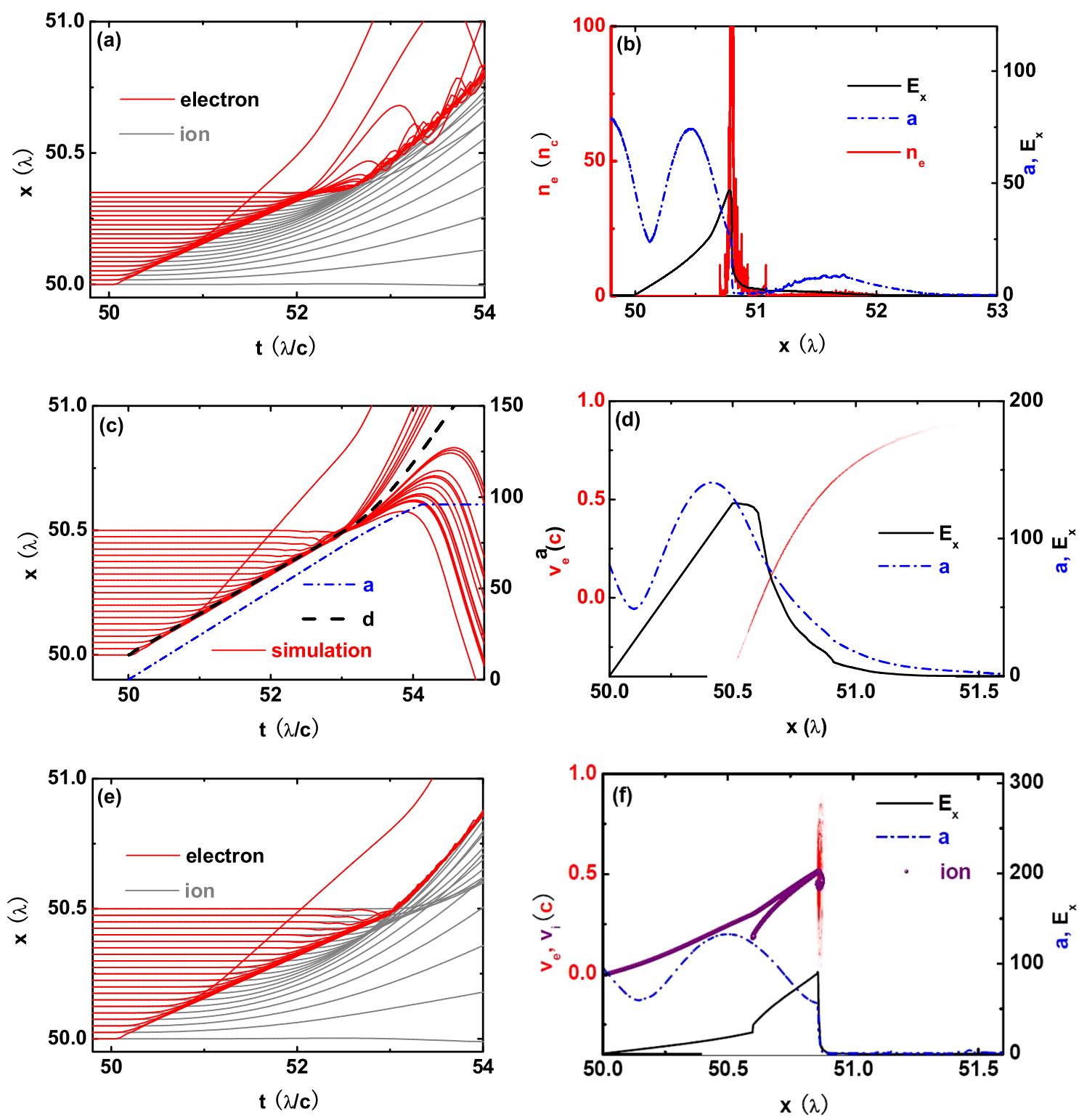

FIG. 5. 1D simulations for (a) and (b) $a_{0}=50$ and $l_{0}=0.35 \lambda$, and (c)-(f) $a_{0}=96$ and $l_{0}=0.5 \lambda$. Here $a_{0} / t_{\text {up }}=29$ and $n_{0}=$ $40 n_{\mathrm{c}}$. The ions are fixed in (c) and (d), and moving in (a), (b), (e), and (f). Red solid lines in (a), (c), and (e) denote electron trajectories. Gray solid lines in (a) and (e) denote ion trajectories. CEL positions (dashed line) and incident laser amplitudes on the CEL surface $a$ (dash dotted line) from the analytical model are plotted in (c). Phase-space distribution of electrons at 54T for (d) fixed and (f) moving ions (purple circles) are shown. $E_{x}$ (black solid line) and $a$ (blue dash dotted line) in simulation are plotted.

$l_{0}=d_{\mathrm{p}}+\delta d=0.5 \lambda$ based on Eq. (16), indicating that a stable CEL state cannot be built if the ions are fixed, as shown in Figs. 5(c) and 5(d). Therefore, Eq. (16) should be used in this case. However, similar to the case in Fig. 5(a), the CEL can still be formed if the fastest ions initially rest in the middle of the foil and the CEL arrives at its back surface simultaneously [see Fig. 5(e)]. The main reason for this condition is that the electrostatic charge-separation field $E_{0}$ is reduced by the rejoining of ions into the CEL [see Figs. 5(d) and 5(f)]. Thus, the laser pressure force on the CEL can be rebalanced by the reduced total electrostatic force.

\section{2D SIMULATIONS}

2D PIC simulations are also carried out to verify the 1D analytical model. In the transverse direction, the laser pulse is four-order super Gaussian with $I=I_{\mathrm{L}} \exp \left[-\left(r / r_{0}\right)^{4}\right]$ and $r_{0}=10 \lambda$. In this case, the focal spot size of the laser is much larger than the foil thickness, thus the transverse escape of electrons can be neglected and the density of the CEL during the hole-boring stage in the 2D simulation is almost identical to that in the 1D case. The 1D model may then be applied. The simulation box is of $100 \mu \mathrm{m}(8000$ cells) in the $x$ direction and $60 \mu \mathrm{m}$ (4800 cells) in the $y$ 

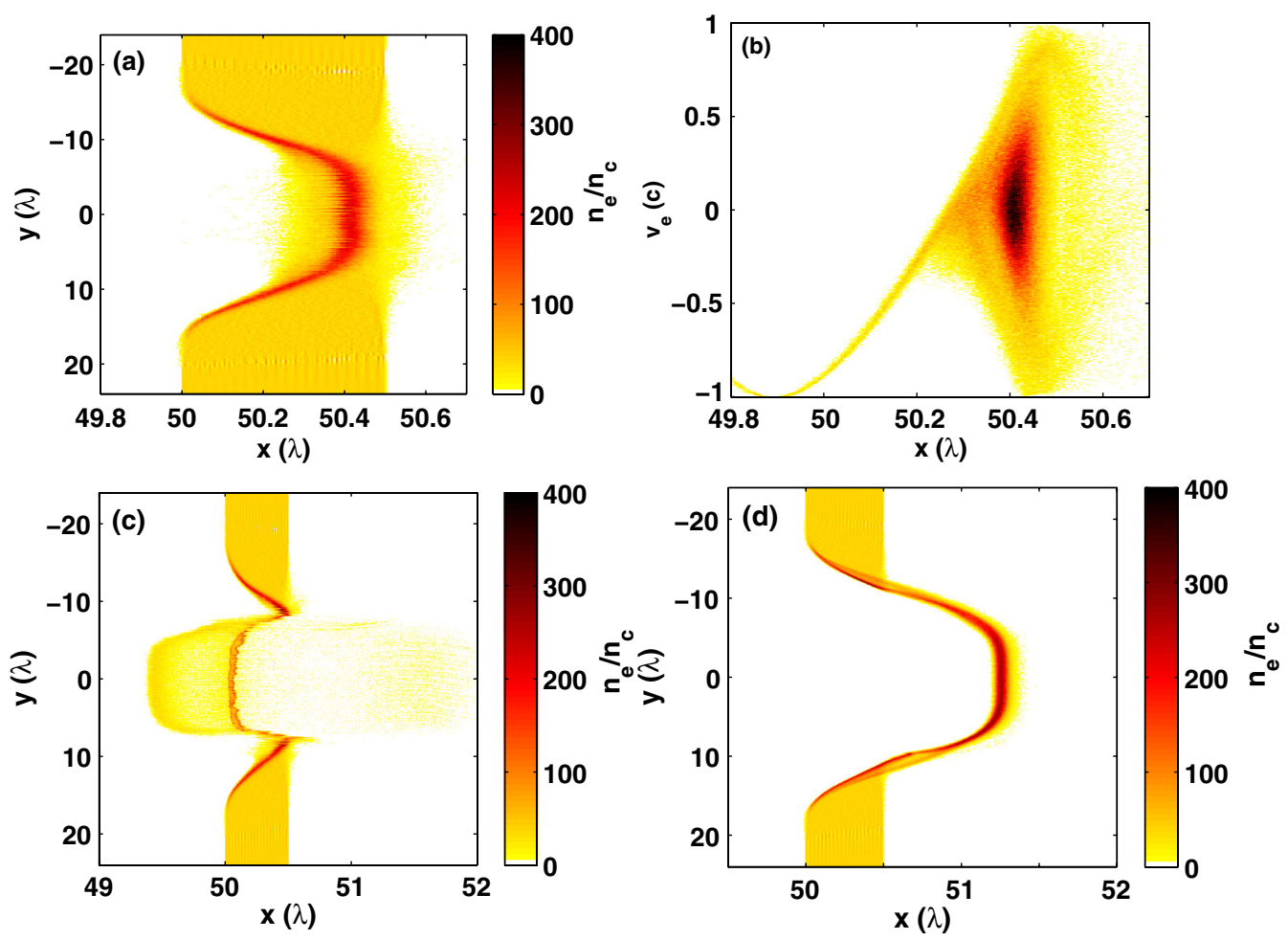

FIG. 6. 2D simulation results for $n_{0}=40 n_{\mathrm{c}}, l_{0}=0.5 \lambda$. (a) Electron-density distributions and (b) phase-space distribution for the electrons in the region $|y| \leq 5 \lambda$ at $t=55 T$ are plotted. Here, $a_{0} / t_{\text {up }}=29\left(a_{0}=50, t_{\text {up }}=1.72 T\right)$. Distributions of electron density at $t=55 T$ for (c) fixed and (d) moving ions for $a_{0} / t_{\text {up }}=29\left(a_{0}=96, t_{\text {up }}=3.3 T\right)$ are plotted.

direction. Each cell is filled with 10 electrons and 10 protons. The laser travels along the $x$ direction. Absorbing boundaries are used for both electromagnetic waves and macroparticles.

First, the case of thick foil is carried out in the 2D simulation. The parameters of the foil and the temporal shape of the laser pulse are identical to those in Fig. 2. Figure 2(b) shows that the position of the CEL during the hole-boring stage is almost consistent with the $1 \mathrm{D}$ analytical model. The CEL is stably located at about $x=50.4 \lambda$ as shown at $t=55 T$ in Fig. 6(a). The corresponding phasespace distribution of electrons is shown in Fig. 6(b), where most of the electrons concentrate around $v_{\mathrm{e}}=0 \mathrm{c}$ at about $x=50.4 \lambda$, consistent with the analytical model and 1D simulations in Figs. 2(a), 2(b), and 2(d). However, this state cannot be kept as stable as that in the 1D case [see Fig. 2(b)], because transverse Rayleigh-Taylor (RT)-like instability slightly develops at $t=55 T$ in Fig. 6(a). Some filaments appear at the front surface of the CEL, which may further modulate the transverse shape of the laser front. The CEL will then completely disperse within several laser periods. In the following discussion, we show that this instability can be reduced somewhat by ion dynamics.

Figures 6(c) and 6(d) show the electron-density distributions at $t=55 T$ for fixed and moving ions, respectively. The CEL is maintained well in the light-sail stage if the fastest ions initially rest in the middle of the foil and the
CEL arrives at the back surface of the foil simultaneously, consistent with the expectation from the 1D analytical model from Eqs. (2)-(5), (17), and (18) and 1D simulation in Fig. 5(e). On the contrary, CEL disperses in the vacuum for the fixed ions [see Fig. 6(c)]. The main reason for this condition is that the rejoining of ions reduces the electrostatic charge-separation field, resulting in the laser pressure force on the CEL being rebalanced by the reduced total electrostatic force. In addition, the transverse RT-like instability in Fig. 6(a) can also be suppressed, to a certain extent, in the case of Fig. 6(d), because the PSA begins as early as possible in the light-sail stage. Therefore some multidimensional instabilities, such as RT and Weibel instability, develop slowly because they grow $\gamma$ times slower in the relativistic frame $[14,23,28]$.

The transverse effect is a challenge in multidimensional [2D or three-dimensional (3D)] cases. The comparison between Figs. 4(a) and 4(b) shows that 2D results have better consistency compared with the 1D model for the sharper laser front. For the smooth laser front, such as $a_{0} / t_{\text {up }}=1$, the CEL disperses long before it reaches the back surface of the foil [see Fig. 4(b)]. This phenomenon is mainly attributed to the fact that multidimensional instabilities may seriously develop with interaction time. Thus, using a laser pulse with a sharp laser front may be a feasible way of suppressing transverse instabilities in multidimensional cases, as shown in Figs. 4(a) and 6(d). In addition, 
the foil thickness should be slightly thicker than $l_{0}=$ $d_{\mathrm{p}}+\delta d$ when using Eq. (16) so that foil deformation by hole boring can be compensated, similar to the case in Fig. 6(a). In this paper, the thin foil irradiated by the intense laser pulse with a sharp front is considered, and the time for hole boring is short such that the multidimensional instabilities and the formation of filamentation can be suppressed to a certain extent. 3D simulation is not shown.

2D simulations for a fully ionized carbon foil irradiated by a CP pulse with intensity $I_{0}=1.1 \times 10^{21} \mathrm{~W} / \mathrm{cm}^{2}$ $\left(a_{0}=20\right)$ were performed to prove the practical utility of our model. In Eq. (2) $a_{t}$ is given by $a_{t}=a_{0} \exp \left(-r^{2} / r_{0}^{2}\right) \sin ^{2}(\pi t / 2 \tau)\left[\sin \left(\omega_{\mathrm{L}} t\right) \hat{\mathbf{e}}_{y}+\cos \left(\omega_{\mathrm{L}} t\right) \hat{\mathbf{e}}_{z}\right]$, where $\tau=7 T$ is the pulse duration and $r_{0}=10 \lambda$. The pulse shape in the transverse direction is Gaussian in nature. The foil is initially located between $x=50 \lambda$ and $50.45 \lambda$, and its electron density is $n_{\mathrm{e}}=15 n_{\mathrm{c}}\left(n_{\mathrm{i}}=2.5 n_{\mathrm{c}}\right.$ for $\mathrm{C}^{6+}$ ). The foil thickness in this case is slightly higher than $l_{0}=0.4 \lambda$ that calculated from Eqs. (2)-(5), (17), and (18) to compensate for foil deformation by hole boring [see Figs. 7(a) and 7(b)]. Figures 7(a)-7(f) show the density distributions of electron $\left(n_{\mathrm{e}}\right)$ and $\mathrm{C}^{6+}\left(n_{\mathrm{i}}\right)$ at $t=54 T$, $56.7 T$, and $65 T$. First, the CEL is driven into the foil leaving ions behind [see Figs. 7(a) and 7(d)]. Both CEL and ions initially rest in the middle of the foil and reach the back side of the foil $(x=50.4 \lambda)$ at about $t=56.7 T$ [see Figs. 7(b) and 7(e)], consistent with the expectation from our model. The laser amplitude acting on the CEL during this time is $a_{t}=19.6(r=0 \lambda)$. Figure $7(\mathrm{~g})$ shows that our model can perfectly describe CEL motion during the
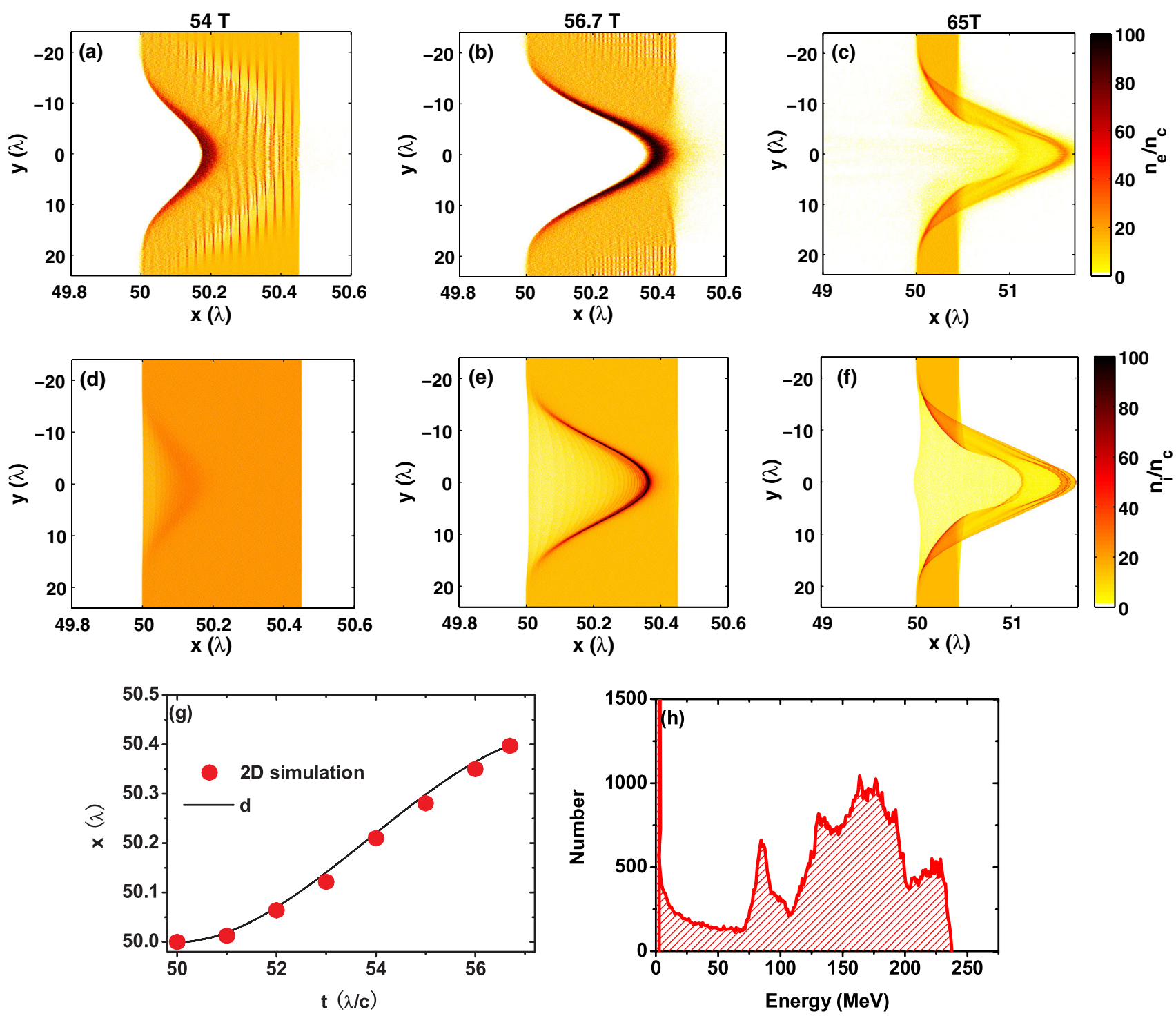

FIG. 7. (a)-(f) $2 \mathrm{D}$ simulation results for electron $\left(n_{\mathrm{e}}\right)$ and $\mathrm{C}^{6+}$ density at $t=54 T, 56.7 T$, and $65 T$. A fully ionized carbon foil is irradiated by the CP laser pulse at intensity $I_{0}=1.1 \times 10^{21} \mathrm{~W} / \mathrm{cm}^{2}\left(a_{0}=20\right)$, where the foil electron density is $15 n_{\mathrm{c}}$ and the foil thickness is $0.45 \lambda$. (g) CEL position $d$ obtained from the model (solid line) and 2D simulation (solid circle). (h) Energy spectra at $t=65 T$ for $\mathrm{C}^{6+}$ ions in the region $|y|<2 \mu \mathrm{m}$. 
hole-boring stage. After hole boring, CEL and ions can be driven together in the light-sail stage. At $t=65 T$, a $\mathrm{C}^{6+}$ beam with a peak energy at about $170 \mathrm{MeV}$ is obtained and the laser pulse is reflected.

\section{CONCLUSIONS}

In summary, a simple 1D time-dependent model is proposed to investigate CEL dynamics in the opacity case. This model can describe interaction details for any shape of laser pulse during sharp-front laser interaction. Expectations from the analytical model can be well verified by $1 \mathrm{D}$ and partly by 2D PIC simulations. The relaxation distance and corresponding relaxation time determined by the laser-front steepness were necessary for stable CEL state formation in the opacity case. In the transparent case, formation of the stable CEL state depends not only on the laser peak amplitude $a_{0}$ but also on the laser-front steepness $a_{0} / t_{\text {up }}$. Compared with the case of fixed ions, laser pressure and electrostatic chargeseparation force can be rebalanced by ions rejoining the CEL as the hole-boring stage changes to the light-sail stage. 2D PIC simulations showed that a $\mathrm{C}^{6+}$ beam with peak energy at about $170 \mathrm{MeV}$ can be obtained by a CP laser pulse at an intensity of $1.1 \times 10^{21} \mathrm{~W} / \mathrm{cm}^{2}$. It should be noted that our model is proposed to describe the CEL dynamics for the interaction of ultrathin foils with intense laser pulses with sharp front. If laser intensity is low or the laser front is too smooth, RPA will be less efficient because the time for hole boring is long enough for the transverse instabilities to develop in the unrelativistic frame. For example, we have performed the simulation when a CP laser pulse at an intensity $I_{0}=2.74 \times 10^{20} \mathrm{~W} / \mathrm{cm}^{2}$ $\left(a_{0}=10\right) . a_{t}=a_{0} \exp \left(-r^{2} / r_{0}^{2}\right) \sin ^{2}(\pi t / 2 \tau)\left[\sin \left(\omega_{\mathrm{L}} t\right) \hat{\mathbf{e}}_{y}+\right.$ $\left.\cos \left(\omega_{\mathrm{L}} t\right) \hat{\mathbf{e}}_{z}\right]$, where $\tau=13 T$ and $r_{0}=10 \lambda$. The foil thickness is $0.48 \lambda$ and electron density is $n_{e}=7 n_{c}$. In this case, the laser front is too smooth or the laser intensity is not high enough, thus the transverse instabilities develop seriously in the unrelativistic hole-boring stage. The electron and ion layer is destroyed during the acceleration process. Fortunately, it is reported recently that the laser with sharp front can be produced by the plasma lens [29]. In addition, $a_{0}>100$ within a rise of $10 \mathrm{fs}$ is highly demanding and may be realized in Extreme Light Infrastructure. So, our results provide a reference for the future experiments. However, a Gaussian pulse with 20 fs FWHM duration rises from $a_{0}=1$ to 10 over $25 \mathrm{fs}$, or around 10 cycles is interesting in experiments. Unfortunately, the effective RPA may be destroyed by the multidimensional instabilities if laser intensity is low.

\section{ACKNOWLEDGMENTS}

This work was supported by the 973 Program (Project No. 2011CB808104), National Natural Science Foundation of China (Projects No. 61008010, No. 11125526,
No. 10834008, and No. 60921004), Shanghai Natural Science Foundation (No. 10ZR1433800), and the Program of Shanghai Subject Chief Scientist (No. 09XD1404300).

[1] V. Chvykov, P. Rousseau, S. Reed, G. Kalinchenko, and V. Yanovsky, Opt. Lett. 31, 1456 (2006).

[2] V. Yanovsky, V. Chvykov, G. Kalinchenko, P. Rousseau, T. Planchon, T. Matsuoka, A. Maksimchuk, J. Nees, G. Cheriaux, G. Mourou, and K. Krushelnick, Opt. Express 16, 2109 (2008).

[3] M. Tabak, J. Hammer, M. E. Glinsky, W. L. Kruer, S. C. Wilks, J. Woodworth, E. M. Campbell, M. D. Perry, and R. J. Mason, Phys. Plasmas 1, 1626 (1994).

[4] N. Naumova, T. Schlegel, V. T. Tikhonchuk, C. Labaune, I. V. Sokolov, and G. Mourou, Phys. Rev. Lett. 102, 025002 (2009).

[5] S. V. Bulanov, T.Z. Esirkepov, V.S. Khoroshkov, A. V. Kuznetsov, and F. Pegoraro, Phys. Lett. A 299, 240 (2002).

[6] S. V. Bulanov and V.S. Khoroshkov, Plasma Phys. Rep. 28, 453 (2002).

[7] M. Borghesi, D. H. Campbell, A. Schiavi, M. G. Haines, O. Willi, A.J. MacKinnon, P. Patel, L. A. Gizzi, M. Galimberti, R. J. Clarke, F. Pegoraro, H. Ruhl, and S. Bulanov, Phys. Plasmas 9, 2214 (2002).

[8] B.F. Shen and Z.Z. Xu, Phys. Rev. E 64, 056406 (2001).

[9] T. Esirkepov, M. Borghesi, S. V. Bulanov, G. Mourou, and T. Tajima, Phys. Rev. Lett. 92, 175003 (2004).

[10] A. Macchi, F. Cattani, T. V. Liseykina, and F. Cornolti, Phys. Rev. Lett. 94, 165003 (2005).

[11] A. P. L. Robinson, M. Zepf, S. Kar, R. G. Evans, and C. Bellei, New J. Phys. 10, 013021 (2008).

[12] X. Q. Yan, C. Lin, Z. M. Sheng, Z. Y. Guo, B. C. Liu, Y. R. Lu, J.X. Fang, and J.E. Chen, Phys. Rev. Lett. 100, 135003 (2008).

[13] X. Zhang, B. Shen, X. Li, Z. Jin, F. Wang, and M. Wen, Phys. Plasmas 14, 123108 (2007).

[14] B. Qiao, M. Zepf, M. Borghesi, and M. Geissler, Phys. Rev. Lett. 102, 145002 (2009).

[15] M. Chen, A. Pukhov, T. P. Yu, and Z. M. Sheng, Phys. Rev. Lett. 103, 024801 (2009).

[16] A. Macchi, S. Veghini, and F. Pegoraro, Phys. Rev. Lett. 103, 085003 (2009).

[17] X.Q. Yan, H.C. Wu, Z.M. Sheng, J.E. Chen, and J. Meyer-ter-Vehn, Phys. Rev. Lett. 103, 135001 (2009).

[18] A. Henig, S. Steinke, M. Schnürer, T. Sokollik, R. Hörlein, D. Kiefer, D. Jung, J. Schreiber, B. M. Hegelich, X. Q. Yan, J. Meyer-ter-Vehn, T. Tajima, P. V. Nickles, W. Sandner, and D. Habs, Phys. Rev. Lett. 103, 245003 (2009).

[19] S. V. Bulanov, E. Y. Echkina, T.Z. Esirkepov, I. N. Inovenkov, M. Kando, F. Pegoraro, and G. Korn, Phys. Rev. Lett. 104, 135003 (2010).

[20] T.-P. Yu, A. Pukhov, G. Shvets, and M. Chen, Phys. Rev. Lett. 105, 065002 (2010). 
[21] V. K. Tripathi, C.S. Liu, X. Shao, B. Eliasson, and R.Z. Sagdeev, Plasma Phys. Controlled Fusion 51, 024014 (2009).

[22] A. P. L. Robinson, Phys. Plasmas 18, 056701 (2011).

[23] W. P. Wang, B.F. Shen, X. M. Zhang, L. L. Ji, M. Wen, J. C. Xu, Y.H. Yu, Y.L. Li, and Z.Z. Xu, Phys. Plasmas 18, 013103 (2011).

[24] A.P.L. Robinson, D. Neely, P. McKenna, and R. G. Evans, Plasma Phys. Controlled Fusion 49, 373 (2007).
[25] F. Cattani, A. Kim, D. Anderson, and M. Lisak, Phys. Rev. E 62, 1234 (2000).

[26] V. I. Eremin, A. V. Korzhimanov, and A. V. Kim, Phys. Plasmas 17, 043102 (2010).

[27] C. S. Lai, Phys. Rev. Lett. 36, 966 (1976).

[28] F. Pegoraro and S. V. Bulanov, Phys. Rev. Lett. 99, 065002 (2007).

[29] H. Y. Wang, C. Lin, Z. M. Sheng, B. Liu, S. Zhao, Z. Y. Guo, Y. R. Lu, X. T. He, J.E. Chen, and X. Q. Yan, Phys. Rev. Lett. 107, 265002 (2011). 